\title{
Metabolomic profiling of breast tumors using ductal fluid
}

\author{
LUISA MATOS DO CANTO $^{1 *}$, CATALIN MARIAN $^{2,3 *}$, RENCY S. VARGHESE $^{1}$, JAEIL AHN $^{1,4}$, \\ PATRICIA A. DA CUNHA ${ }^{1}$, SHAWNA WILLEY ${ }^{1,5}$, MARY SIDAWY ${ }^{1,6}$, JANICE D. RONE ${ }^{1}$, AMRITA K. CHEEMA ${ }^{1,7}$, \\ GEORGE LUTA $^{1,4}$, MOHAMMAD R. NEZAMI RANJBAR ${ }^{1}$, HABTOM W. RESSOM ${ }^{1}$ and BASSEM R. HADDAD ${ }^{1}$
}

\author{
${ }^{1}$ Lombardi Comprehensive Cancer Center and Department of Oncology, Georgetown University Medical Center, Georgetown \\ University, Washington DC, USA; ${ }^{2}$ Biochemistry Department, 'Victor Babes' University of Medicine and Pharmacy, \\ Timisoara, Romania; ${ }^{3}$ Ohio State University Comprehensive Cancer Center, The Ohio State University, Columbus, OH; \\ ${ }^{4}$ Department of Biostatistics, Bioinformatics and Biomathematics, Georgetown University Medical Center, Georgetown \\ University, Washington DC; Departments of ${ }^{5}$ Surgery and ${ }^{6}$ Pathology, MedStar Georgetown University Hospital, \\ Georgetown University, Washington DC; ${ }^{7}$ Department of Biochemistry and Molecular and Cellular Biology, \\ Georgetown University Medical Center, Georgetown University, Washington DC, 20007, USA
}

Received July 15, 2016; Accepted September 26, 2016

DOI: $10.3892 /$ ijo.2016.3732

\begin{abstract}
Identification of new biomarkers for breast cancer remains critical in order to enhance early detection of the disease and improve its prognosis. Towards this end, we performed an untargeted metabolomic analysis of breast ductal fluid using an ultra-performance liquid chromatography coupled with a quadrupole time-of-light (UPLC-QTOF) mass spectrometer. We investigated the metabolomic profiles of breast tumors using ductal fluid samples collected by ductal lavage (DL). We studied fluid from both the affected breasts and the unaffected contralateral breasts (as controls) from 43 women with confirmed unilateral breast cancer. Using this approach, we identified 1560 ions in the positive mode and 538 ions in the negative mode after preprocessing of the UPLC-QTOF data. Paired t-tests applied on these data matrices identified 209 ions (positive and negative modes combined) with significant change in intensity level between affected and unaffected control breasts (adjusted P-values <0.05). Among these, 83 ions (39.7\%) showed a fold change (FC) $>1.2$ and 66 ions $(31.6 \%)$ were identified with putative compound names. The metabolites that we identified included endogenous metabolites such as amino acid derivatives (N-Acetyl-DLtryptophan) or products of lipid metabolism such as N-linoleoyl taurine, trans-2-dodecenoylcarnitine, lysophosphatidylcholine LysoPC(18:2(9Z,12Z)), glycerophospholipids PG(18:0/0:0),
\end{abstract}

Correspondence to: Dr Bassem R. Haddad, Lombardi Comprehensive Cancer Center and Department of Oncology, Georgetown University Medical Center, Georgetown University, 3800 Reservoir Road NW, New Research Building E204, Washington DC 20007, USA

E-mail: haddadb1@georgetown.edu

*Contributed equally

Key words: breast cancer, ductal fluid, metabolomics and phosphatidylserine PS(20:4(5Z,8Z,11Z,14Z). Generalized LASSO regression further selected 21 metabolites when race, menopausal status, smoking, grade and TNM stage were adjusted for. A predictive conditional logistic regression model, using the LASSO selected 21 ions, provided diagnostic accuracy with the area under the curve of 0.956 (sensitivity/ specificity of $0.907 / 0.884$ ). This is the first study that shows the feasibility of conducting a comprehensive metabolomic profiling of breast tumors using breast ductal fluid to detect changes in the cellular microenvironment of the tumors and shows the potential for this approach to be used to improve detection of breast cancer.

\section{Introduction}

Breast cancer is one of the most genetically heterogeneous cancers. It is also one of the most commonly diagnosed cancers and the second most common cause of cancer mortality in women (1). Despite the improvement of current screening methods which rely on imaging techniques and the targeted classification of breast cancer based primarily on hormone receptors (estrogen and progesterone) and the HER2/NEU status, mortality remains high for advanced breast cancer, particularly when drug resistance develops.

Since Warburg first hypothesized that cancer cells had a significantly higher rate of glycolysis than normal cells (2), researchers have shown that changes in metabolism can lead to large changes in metabolites that occur downstream of genomic and proteomic alterations $(3,4)$. How signal pathways control energy metabolism in cancer cells has become a high priority in cancer research, reflecting an increased focus on the interaction of gene expression (i.e. receptors), microenvironment (i.e. hypoxia), and glucose metabolism, and how the downstream expression of metabolites can be used for both early detection of breast cancer as well as targeted drug therapies (5-7).

Traditional methods of analysis have evolved into largescale data gathering through different 'omics' approaches: 
genomics, transcriptomics, proteomics and more recently, metabolomics. This latter growing technology permits simultaneous monitoring of thousands of macro and micro metabolites that serve as substrates and products of metabolic pathways (8). Integration of these pathways and their interactions provides insight into the development of malignant processes and could lead to the discovery of cancer biomarkers (9).

Several studies have demonstrated the efficacy of using metabolomics to discriminate between cancer and normal tissues in different organs including the breast. Sitter et al (10) analyzed 88 tumor samples from breast cancer patients and 18 tissue samples from adjacent non-tumor tissue using high-resolution magic-angle spinning magnetic resonance spectroscopy (HRMAS). Principal component analysis (PCA) allowed for correct sample classification in the majority of the cases with $82 \%$ sensitivity and $100 \%$ specificity. Mountford et al (11) performed proton nuclear magnetic resonance ( $\left.{ }^{1} \mathrm{HNMR}\right)$ spectroscopy analysis of breast tumor extracts. Fine needle aspiration biopsies from 140 patients with breast lumps (83 malignant and 57 benign) were analyzed by ${ }^{1} \mathrm{HNMR}$ spectroscopy. Using a classification strategy, they were able to classify samples as malignant or benign with a sensitivity and specificity of 93 and 92\%, respectively. More recently, using high-throughput gas chromatography with time-of-flight mass spectrometer (GC-TOFMS)-based metabolomic analysis, Budczies et al (12) identified significant differences between metabolites from breast tumors compared to normal tissues, specifically the cytidine-5-monophosphate/pentadecanoic acid metabolic ratio. This allowed the discrimination between normal and cancer tissue samples with high specificity (93.9\%) and high sensitivity (94.8\%). Furthermore, a comparison of estrogen receptor positive and estrogen receptor negative breast cancer revealed significant changes in glutamine and $\beta$-alanine metabolism between these two breast cancer subtypes (13). Metabolomic profiling was used to discriminate between localized early breast cancer and advanced metastatic disease (14), and to develop a prediction model for the early detection of recurrent breast cancer from serum samples (15). Of interest, Budhu et al (16), showed that there was a specific metabolomic signature of tumors depending on the tissue of origin and suggested that the metabolites were generally unique for each tissue and cancer type. Comparing the metabolic changes between cancer and normal cells could identify the metabolic reprograming involved in tissue specific tumorigenesis.

To date, metabolomic analysis has been performed on many different tissue types, including solid tissues, serum, plasma and urine (17). Originally, ductal lavage (DL) and nipple aspirate fluid (NAF) were used for cytological evaluation of breast epithelial cells in the ductal fluid. They have also been used for different molecular studies. However, because they contain proteins and metabolites of breast tissue metabolism in addition to ductal epithelial cells, they are very useful for metabolomic studies, thus providing a unique opportunity to evaluate more directly metabolomic changes in the breast tumor microenvironment itself and avoiding questions of tissue specificity, which arise when evaluating blood and urine. The feasibility of performing metabolomic analysis in NAF was recently demonstrated in a small study of eight subjects (18). The study was conducted on samples obtained from healthy pre- and post-menopausal individuals and compared the findings in NAF with matching plasma samples from the same patients. They showed that NAF is metabolically distinct from matched plasma samples which supports the theory that the cellular environment (tumor microenvironment) is more directly mirrored in breast biofluids (DL and NAF). We have recently identified a panel of microRNAs that are differentially expressed in ductal fluid from breasts with tumors compared to paired ductal fluid samples from the contralateral normal breast (19), further substantiating the importance of a more direct analysis of the tumor microenvironment and the potential for biomarker development using ductal fluid obtained in a non-invasive or minimally invasive approach. Here, we report the first metabolomic analysis of breast ductal fluid samples obtained from 43 patients with unilateral breast cancer. We evaluated paired samples from the breast with cancer compared to the contralateral non-affected breast (control) and identified several metabolites with significant changes in levels between affected and non-affected breasts. This approach provides an exciting opportunity to detect metabolomic changes in the cellular microenvironment reflecting tumor evolution, and has the potential for significantly improving breast cancer screening and detection

\section{Materials and methods}

Patient population. We enrolled 43 patients with unilateral, biopsy-confirmed, breast tumors [invasive breast cancer (IBC) and/or ductal carcinoma in situ (DCIS)], who were scheduled for surgery (mastectomy/lumpectomy) at the MedStar Georgetown University Hospital. Patients were identified by the surgeon and offered the opportunity to participate in the study. If they agreed, they were asked to sign an IRB-approved informed consent.

Ductal lavage. Prior to starting the operative procedure, for each subject, the surgeon obtained breast ductal fluid from the affected breast and the non-affected contralateral breast, using ductal lavage. Each patient served as her own control. The ductal lavage procedure was performed as previously described (20), except that the collected fluid was placed in a sterile tube with no preservative solution, and was transferred immediately to the laboratory, and divided into different aliquots which were frozen at $-80^{\circ} \mathrm{C}$ for future studies. One fresh aliquot was used for cytopathology evaluation to investigate the presence of benign, atypical or malignant cells by a certified breast pathologist, using the established criteria for ductal lavage cytologic analysis (21).

Metabolite extraction from ductal lavage. Metabolite extraction was performed as per the protocol described by Sheikh et al (22). Briefly, $150 \mu \mathrm{l}$ of ductal lavage fluid was plunged into dry ice for $30 \mathrm{sec}$ followed by heat shock at $37^{\circ} \mathrm{C}$ for $30 \mathrm{sec}$. A total of $600 \mu \mathrm{l}$ of methanol containing 4-nitrobenzoic acid and debrisoquine were then added and the samples were vortexed, transferred to room temperature and extracted with chloroform. The tubes were transferred to $-20^{\circ} \mathrm{C}$ for overnight incubation and subsequently centrifuged at $4^{\circ} \mathrm{C}$ for $10 \mathrm{~min}$ at $12,000 \mathrm{rpm}$. The top and bottom phases were transferred to different tubes carefully avoiding the middle 
interface (containing precipitated proteins). An equivalent amount of chilled acetonitrile (ACN) was then added and the samples cooled on ice for $15 \mathrm{~min}$ after vortexing. Samples were centrifuged at $4^{\circ} \mathrm{C}$ for $10 \mathrm{~min}$ at $12,000 \mathrm{rpm}$ and the supernatant was transferred to a fresh tube and dried under vacuum. The residual pellet was re-suspended in $200 \mu \mathrm{l}$ of solvent A (98\% water, $2 \% \mathrm{ACN}$ and $0.1 \%$ formic acid) for UPLC-Q-TOF/MS analysis.

UPLC-QTOF data acquisition. Each sample (5 $\mu \mathrm{l})$ was injected onto a reverse-phase $50 \times 2.1 \mathrm{~mm}$ BEH $1.7 \mu \mathrm{m}$ C18 column using an Acquity UPLC system (Waters Corp., Milford, MA, USA). The mobile phase comprised of water containing $0.1 \%$ formic acid solution (A) and acetonitrile containing $0.1 \%$ formic acid solution (B). Each sample was resolved for $10 \mathrm{~min}$ at a flow rate of $0.5 \mathrm{ml} / \mathrm{min}$. This approach has been extensively used for metabolomic profiling of biofluids; UPLC gradient conditions and the mass spectrometry parameters have been described in detail $(23,24)$. The column eluent was introduced directly into the mass spectrometer by electrospray. Mass spectrometry was performed on a Quadrupole-time-of-flight mass spectrometer operating in either negative or positive electrospray ionization mode with a capillary voltage of $3.2 \mathrm{kV}$ and a sampling cone voltage of $35 \mathrm{~V}$. The desolvation gas flow was $800 \mathrm{l} / \mathrm{h}$ and the temperature was set to $350^{\circ} \mathrm{C}$. The cone gas flow was $50 \mathrm{l} / \mathrm{h}$, and the source temperature was $150^{\circ} \mathrm{C}$. Accurate mass was maintained by infusing sulfadimethoxine $(311.0814 \mathrm{~m} / \mathrm{z})$ in $50 \%$ aqueous acetonitrile $(250 \mathrm{pg} / \mu \mathrm{l})$ at a rate of $30 \mu \mathrm{l} / \mathrm{min}$ via the lockspray interface every $10 \mathrm{sec}$. Data were acquired in centroid mode from 50 to $850 \mathrm{~m} / \mathrm{z}$ mass range for TOF-MS scanning, in duplicate (technical replicates) for each sample in positive and negative ionization mode and checked for chromatographic reproducibility. For all profiling experiments, the sample queue was staggered by randomizing samples to eliminate bias. We acquired UPLC-QTOF data by analysis of DL from 43 subjects. For each subject two samples, one from the affected and one from the contralateral normal breast, were generated. Each sample was injected twice.

Data preprocessing. The raw UPLC-QTOF data were converted into Network Common Data Format (NetCDF) using the MassLynx software (Waters Corp.). The R-package XCMS (Scripps Center for Metabolomics, La Jolla, CA, USA) was used to preprocess the datasets acquired in the electrospray positive and negative ion modes. The first step in XCMS is to detect the peaks. The peak detection algorithm first cuts the data into slices, a fraction of a mass unit wide, and then applies a model peak matched filter on those individual slices over the chromatographic time domain. After detecting peaks in individual samples, the peaks are matched across samples to allow calculation of retention time (RT) deviations and relative ion intensity comparison. This is accomplished using a grouping method that uses kernel density estimation to group peaks in the mass domain. The peak matching algorithm in XCMS takes into account the two-dimensional anisotropic nature of data. These groups are then used to identify and correct drifts in RT from run to run.

Following peak matching, we used the R-package CAMERA to identify derivative ions originating from the same compound in the form of adducts, isotopes, and in-source fragments (25). Adducts of a molecule are formed during the electrospray ionization (ESI) process, e.g. sodium and ammonium adduct. Ions of molecular isotopes are detected with distinct $\mathrm{m} / \mathrm{z}$ values during $\mathrm{MS}$ analysis and the peak with the lowest $\mathrm{m} / \mathrm{z}$ is defined as the monoisotopic peak. In-source fragments are formed during ionization such as ion fragments of $\left[\mathrm{M}+\mathrm{H}-\mathrm{H}_{2} \mathrm{O}\right]^{+}$or $\left[\mathrm{M}-\mathrm{H}-\mathrm{H}_{2} \mathrm{O}\right]^{-}$through neutral loss of water molecule. Different adducts/isotopes/water-loss products of the same compound theoretically share the same retention time in chromatograms. As long as the scan rate is properly adjusted and enough scanning points are acquired to define the chromatographic peaks, the ions from the same compound share similar-shaped elution profiles which can be represented by their extracted ion chromatograms (EICs). Thus, clustering of similar elution profiles was performed by CAMERA prior to statistical analysis. Recognition of such metabolites, often represented by multiple peaks with distinct $\mathrm{m} / \mathrm{z}$ values at similar retention times, can facilitate metabolite identification in LC-MS based metabolomics.

Statistical analysis. To identify ions with significant changes in intensity levels, we used parametric statistical methods that we implemented in-house using MATLAB (MathWorks, Natick, MA, USA) and R scripts. Before performing the statistical analysis, intensities from double injections were averaged to achieve a single intensity for each right or left DL sample. For pre-screening of ions, both univariate analyses and multivariate analyses were performed. For each ion, paired t-tests were performed to compare profiles of metabolites between the tumor samples and the paired normal samples. P-values obtained from multiple testing were adjusted to q-values based on Storey's method (26). A robust pairwise fold change (FC) was calculated based on the median of the relative intensity of tumor/normal samples for each pair. In addition, we performed principal component analysis (PCA) and multilevel PLS-DA (MPLS-DA) that accounts for the paired data (27). The first screening was performed based on q-values $<0.05$. To further identify which ions are associated with the normal/tumor tissues, generalized LASSO regression adjusting for race, menopausal status, smoking, grade, and TNM stage and conditional logistic regression with LASSO penalties were fitted where LASSO penalty parameters were determined through 10-fold cross-validation (using the 'glmnet' and 'clogitL1' R package, respectively) $(28,29)$. Conditional logistic regression with the selected ions was employed as a predictive model where the diagnostic performance of the selected ions was assessed using the area under the receiver operating characteristic (ROC) curves. In addition, one way ANOVA and linear regression were used to compare cancer metabolomic profiles of LASSO selected ions across different demographic and clinical characteristics.

Putative identifications for the resulting ion list were obtained through a mass-based search using MetaboSearch (30), which searches for putative identifications against four databases: the Human Metabolite DataBase (HMDB) (31), Metlin (32), Madison Metabolomics Consortium Database (MMCD) (33), and LIPID MAPS (34). The mass tolerance in the database search was set to $10 \mathrm{ppm}$. The $\mathrm{m} / \mathrm{z}$ values of annotated isotopes/adducts/in-source fragments peaks were converted to the corresponding neutral mono-isotopic masses 
Table I. Subject characteristics.

\begin{tabular}{lll}
\hline Characteristics & $\mathrm{N}$
\end{tabular}

\begin{tabular}{lc}
\hline Age $($ mean \pm SD) & $52.20( \pm 12.25)$ \\
Menopause & \\
Pre & 18 \\
Post & 25
\end{tabular}

Race/Ethnicity

A
CA
H

Family history of breast cancer

Yes

$4 \quad 9.30$

$11 \quad 25.59$

$26 \quad 60.46$

$2 \quad 4.65$

No

Tumor site

Right

16

Left

27

Smoking history

Current

Former

Never

Histological type

DCIS
IDC
ILC
Mixed

Stage

$\begin{array}{lr}0 & 77 \\ \text { I } & 17 \\ \text { II } & 18 \\ \text { III } & 1 \\ \text { Grade } & \end{array}$

rade

Low

Intermediate

High

41.86

58.14

9.30

4.46
4.65

41.86

58.14

37.21

62.79

9.30

16.28

74.42

16.28

72.10

9.30

2.32

16.28

39.54

41.86

2.32

6.98

32.56

60.46

Lymph node involvement

Yes

30.23

No

13

29

ER

Positive

38

Negative

5

PR

Positive

$$
30
$$

Negative

HER2

Positive

Negative

8

30

Affected breast cytology

Atypical cells

Benign cells

Insufficient cells
Table II. Number of ions detected and those selected by statistical analysis.

\begin{tabular}{lccc}
\hline Mode & $\begin{array}{c}\text { No. of ions } \\
\text { detected }\end{array}$ & $\begin{array}{c}\text { No. of ions } \\
\text { with adjusted } \\
\text { P-value }<0.05\end{array}$ & $\begin{array}{c}\text { No. of ions selected } \\
\text { by LASSO }\end{array}$ \\
\hline Positive & 1560 & 197 & 19 \\
Negative & 538 & 12 & 2 \\
\hline
\end{tabular}

before searching them against the databases. Identities of a subset of the putative metabolite identifications were verified by comparing their MS/MS fragmentation patterns and RT with those of authentic standard compounds.

\section{Results}

Clinical and tumor characteristics of the patients are summarized in Table I. Our patient population was 52 years of age on average, the majority being post-menopausal (58\%). Approximately $60 \%$ of our patients were white, and a quarter of them were African Americans. The great majority were never or former smokers. Most of the tumors were invasive ductal carcinoma (IDC) (72\%), early stage (I and II) (81\%), with no lymph node involvement $(67 \%)$, hormone receptor positive $\left(88 \% \mathrm{ER}^{+}, 70 \% \mathrm{PR}^{+}\right)$, and HER2 negative $(70 \%)$. More than two thirds of our patients had insufficient cells for analysis in the ductal lavage samples from the affected breast (70\%), emphasizing the limitation of ductal lavage cytology for detecting breast cancer. Subjects who received pre-operative chemotherapy were excluded from the study.

Preprocessing of the UPLC-QTOF data identified 2098 ions, 1560 ions in the positive mode and 538 ions in negative mode (Table II). These data matrices were used to select ions with significant differences between ductal lavage fluid from the affected breast and the non-affected contralateral control breast. Paired t-tests were applied on these data matrices and identified 209 ions, out of the 2,098 ions, with q-values $<0.05$ (Table II). Among the 209 significant ions, 66 (31.6\%) were assigned putative compound names by MetaboSearch. When ranking these ions according to the fold change (FC) in ion intensity levels between the affected and non-affected breasts, $83(39.7 \%)$ metabolites showed an FC $\geq 1.2,37$ of these, being metabolites with a putative compound name identified through the databases. The great majority of these compounds were most likely of exogenous origin, being found in plants, dietary supplements or drugs; however, some were endogenous metabolites such as amino acid derivatives (N-Acetyl-DLtryptophan, $\mathrm{FC}=-2.02, \mathrm{P}=0.006)$ or products of lipid metabolism such as $\mathrm{N}$-linoleoyl taurine $(\mathrm{FC}=-1.24, \mathrm{P}=0.001)$, trans-2-dodecenoylcarnitine $(\mathrm{FC}=-3.58, \mathrm{P}=0.006)$, lysophosphatidylcholine LysoPC(18:2(9Z,12Z)) $(\mathrm{FC}=-1.20, \mathrm{P}=0.018)$, and glycerophospholipids $\mathrm{PG}(18: 0 / 0: 0) \quad(\mathrm{FC}=-1.45, \mathrm{P}=0.018)$ and phosphatidylserine $\mathrm{PS}(20: 4(5 \mathrm{Z}, 8 \mathrm{Z}, 11 \mathrm{Z}, 14 \mathrm{Z})(\mathrm{FC}=-1.49$, $\mathrm{P}<0.001)$. Logistic regression with LASSO penalties further selected 21 metabolites, when race, menopausal status, smoking, grade and TNM stage were adjusted for (Table III). However, conditional logistic regression with LASSO penalties

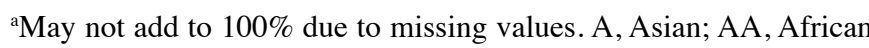
American; CA, Caucasian; H, Hispanic; DCIS, ductal carcinoma in situ; IDC, invasive ductal carcinoma; ILC, invasive lobular carcinoma.

20.93
69.77
African
rcinoma 


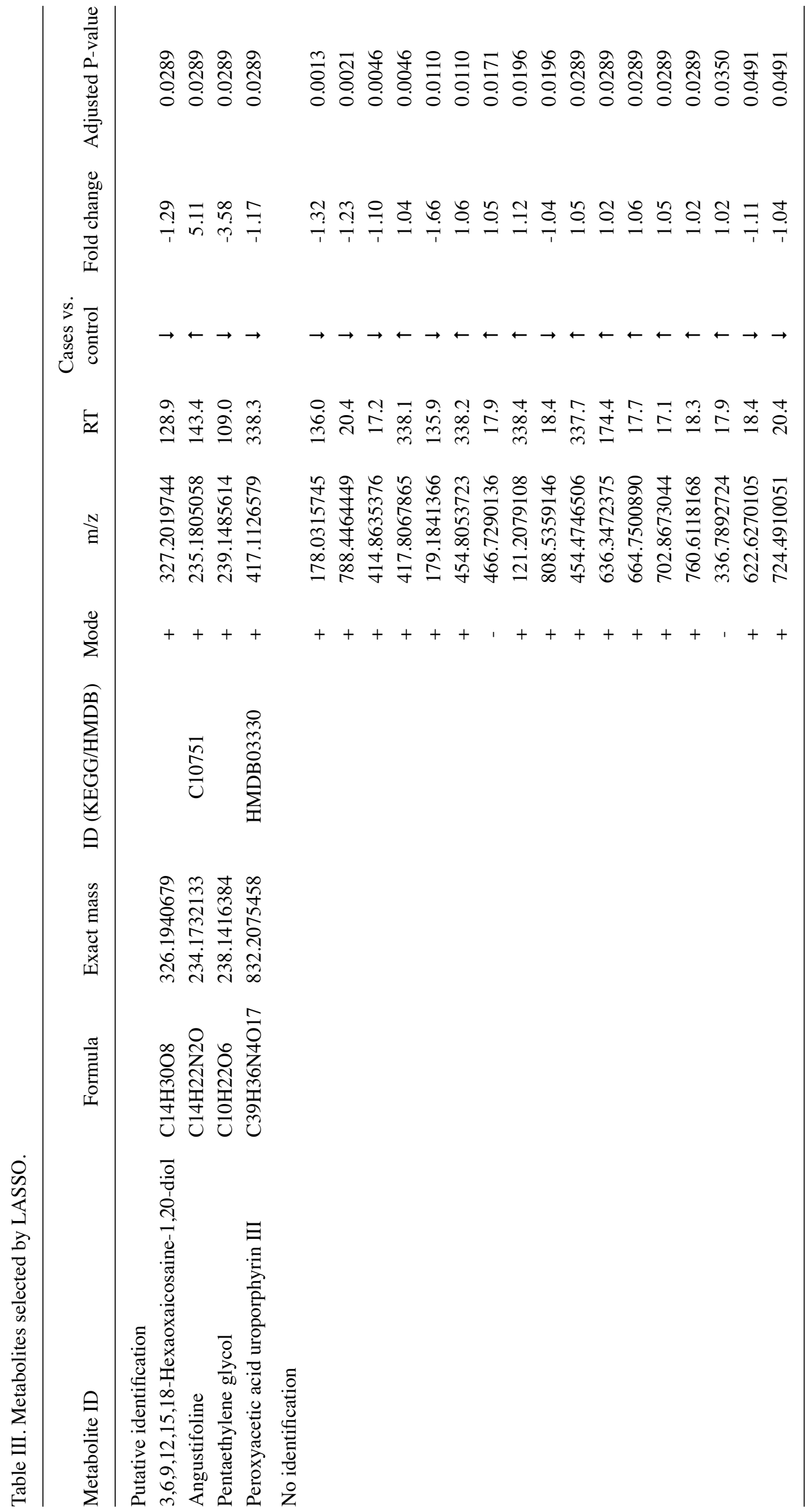



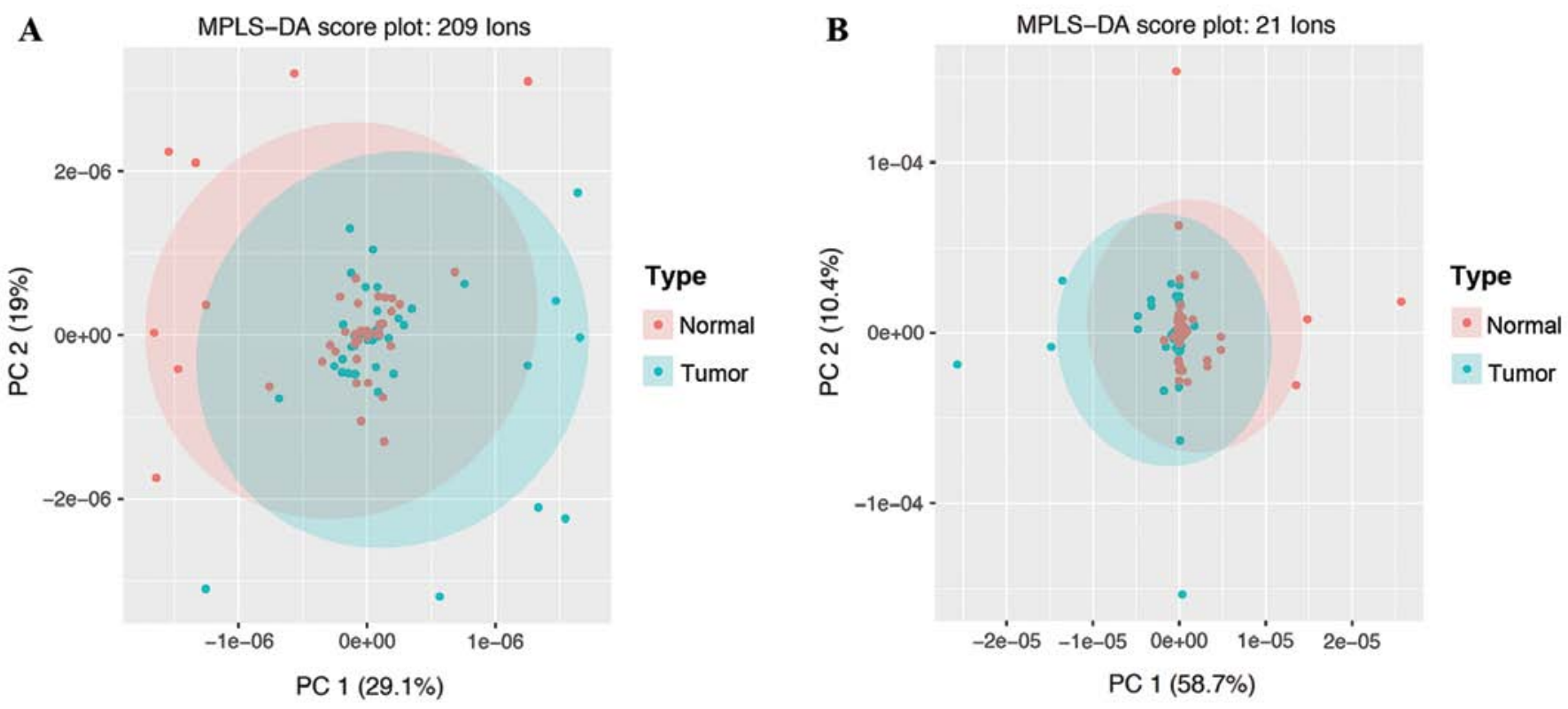

Figure 1. MPLS-DA score plot shows the separation between two class labels (normal and tumor) based on (A) the significant 209 ions with q-value $<0.05$ and (B) the 21 ions that were selected from LASSO regression with the pre-screened 209 ions.

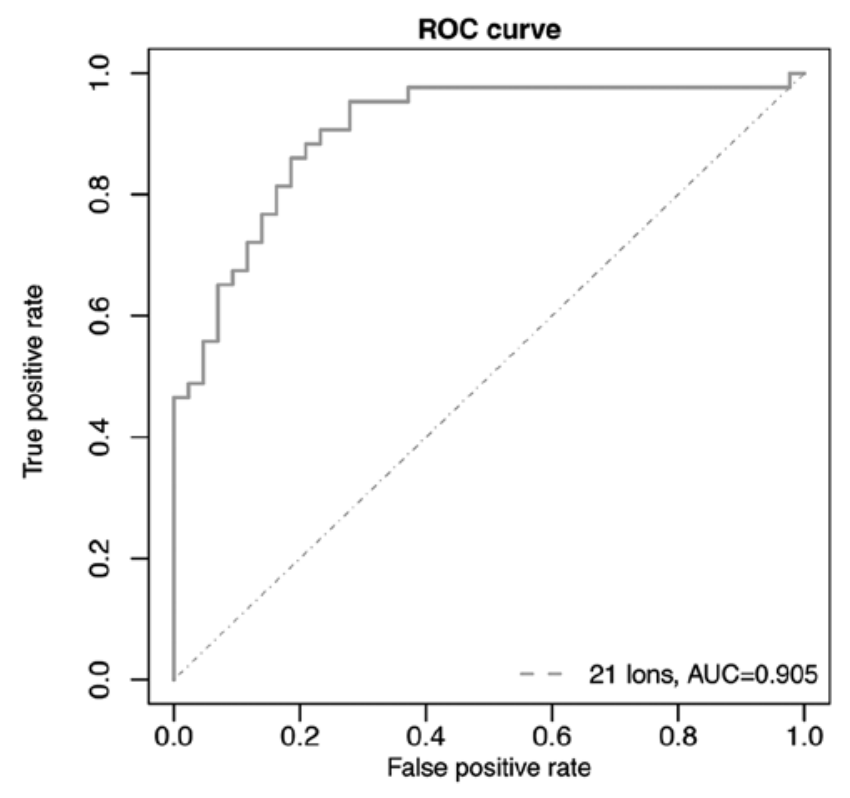

Figure 2. Receiver operating characteristics (ROC) curve with the area under curve (AUC) showing the prediction performance of the 21 selected ions with a sensitivity and specificity of 90.7 and $88.4 \%$, respectively, when the cut-off was set to 0.27 .

yielded five ions where the predictive model does not provide adequate prediction performance (AUC $=0.679$, sensitivity/ specificity of $0.744 / 0.581$ ). Thus, further inferences focused on the 21 ions.

We performed principal component analysis (PCA) and multilevel PLS-DA (MPLS-DA) that accounts for the cross-over data and examined the performance of selected biomarkers. The PCA plot based on the 21 ions (identified using the LASSO regression analysis; Fig. 1B) shows visually better clustering performances of both sets of ions than the PCA plot based on 209 ions (q-values $<0.05$; Fig. 1A).
Table IV. Metabolites, among the LASSO identified ions, that show statistically significant differences based on menopausal status (pre/post), ER (+/-) and HER2 (+/-).

\begin{tabular}{llcll}
\hline $\mathrm{M} / \mathrm{z}$ & \multicolumn{1}{c}{$\mathrm{RT}$} & Menopause & ER & Her2 \\
\hline 239.1486 & 109.0287 & 0.849 & $\mathbf{0 . 0 4 5}$ & 0.437 \\
179.1841 & 135.8769 & $\mathbf{0 . 0 2 6}$ & 0.432 & 0.226 \\
121.2079 & 338.3861 & $\mathbf{0 . 0 4 9}$ & 0.788 & $\mathbf{0 . 0 0 0}$ \\
178.0316 & 136.0036 & 0.124 & 0.3 & $\mathbf{0 . 0 3 6}$ \\
417.1127 & 338.331 & 0.238 & 0.997 & $\mathbf{0 . 0 2 2}$ \\
454.4747 & 337.7336 & 0.538 & 0.388 & $\mathbf{0 . 0 0 3}$ \\
622.627 & 18.4193 & $\mathbf{0 . 0 4}$ & 0.777 & 0.280 \\
724.491 & 20.4252 & 0.269 & 0.407 & $\mathbf{0 . 0 0 4}$ \\
788.4464 & 20.4252 & 0.649 & 0.673 & $\mathbf{0 . 0 0 1}$ \\
466.729 & 17.86 & $\mathbf{0 . 0 2 6}$ & 0.774 & 0.133
\end{tabular}

$\mathrm{P}$-values are in bold when statistically significant at $\mathrm{p}<0.05$.

We evaluated whether the LASSO-selected 21 ions can be used as potential biomarkers for breast cancer detection. Conditional logistic regression with the 21 ions was employed as a predictive model where the diagnostic performance of the 21 ions was assessed using the area under the receiver operating characteristic (ROC) curves. ROC based on the conditional logistic regression model gave highly accurate diagnostic performance with an area under the curve (AUC) of 0.956 along with a sensitivity of $90.7 \%$ and specificity of $88.4 \%$ (Fig. 2). In addition, one way ANOVA and linear regression were used to compare cancer metabolomic profiles of the LASSO-selected ions across different demographic and clinical characteristics. Among the 21 LASSO-identified ions, we found ten metabolites that show statistically significant differences based on menopausal status (pre/post), ER (+/-), 
Table V. The verified and unverified metabolites.

\begin{tabular}{lccclcr}
\hline M/Z & RT(sec) & RT(min) & Monoisotopic mass & Putative name of the compound & FC & q-value \\
\hline Verified metabolites & & & & & & \\
247.1071 & 187 & 3.12 & 247.1070665 & N-Acetyl-Dtryptophan & -2.02309 & 0.049087 \\
134.0966 & 136 & 2.27 & 134.0965659 & $1,2,3,4$-tetrahydroisoquinoline & -1.12989 & 0.049087 \\
179.0568 & 136 & 2.27 & 179.0567725 & Gluconolactone & -1.26445 & 0.049087 \\
513.3184 & 351 & 5.85 & 513.3184402 & Phosphatidyl Glycerol (18:0/0:0) & -1.45152 & 0.049087 \\
174.1487 & 234 & 3.90 & 174.1487168 & 9-amino-nonanoic acid & -1.15122 & 0.028858 \\
433.2574 & 440 & 7.33 & 433.2574371 & Hydrocortisone butyrate & -1.08314 & 0.028858 \\
Unverified metabolites & & & & & & \\
620.5977 & 19 & 0.32 & 620.5977263 & Ceramide (d18:2/22:0) & -1.08974 & 0.028858 \\
429.2271 & 365 & 6.08 & 429.2270941 & Phosphatidyl glycerol (12:0/0:0) & 1.03441 & 0.019619 \\
\hline
\end{tabular}

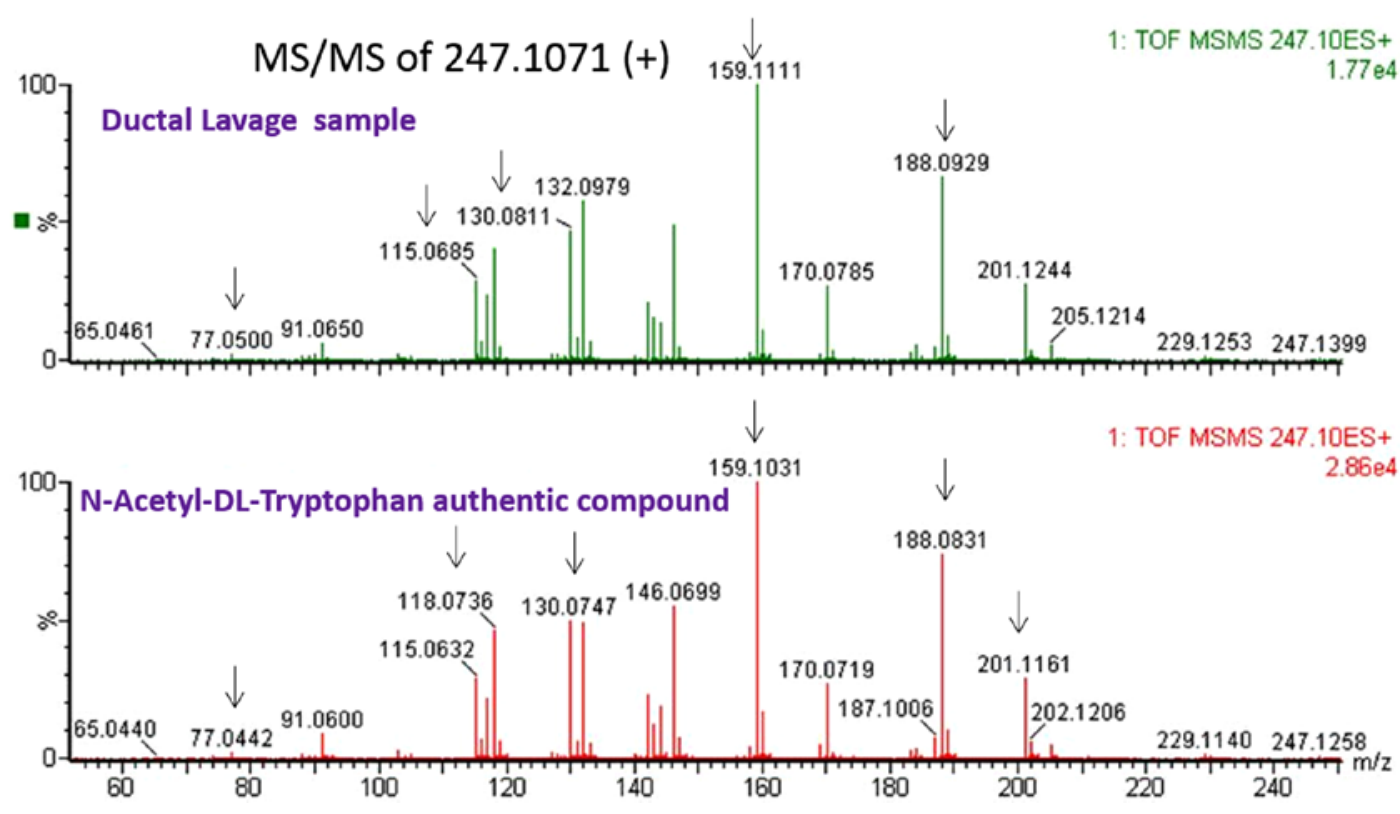

Figure 3. Verification of putative ID with mass 247.1071. Top panel is the MS/MS spectrum of the ion obtained in the ductal lavage samples and bottom panel is the MS/MS spectrum of authentic compound N-Acetyl-DL-tryptophan.

and HER2 (+/-). Table IV shows these metabolites. P-values are in bold for the metabolites with $\mathrm{P}<0.05$.

We found that menopausal status can affect the level of metabolite expression among our patients. Three metabolites (M179.1841, M121.2079 and M466.729) are higher in postmenopausal patients $(\mathrm{P}<0.05$; Table IV). On the other hand, one metabolite (M622.627) is higher in pre-menopausal patients $(\mathrm{P}=0.040$; Table IV). HER2 positive receptor status also shows an association with increased levels of metabolites (M724.491 and M788.4464) in patients $(\mathrm{P}<0.005$; Table IV), while four metabolites (M121.2079, M178.0316, M417.1127 and M454.4747) are lower in HER2 positive patients $(\mathrm{P}<0.05$; Table IV).

From the list of 209 significant monoisotopic ions with putative identifications, we selected eight metabolites with putative IDs and standard compounds for verification by comparing their TOF-MS/MS spectra against their corresponding synthetic compounds. Specifically, we ran an authentic compound for each candidate and a patient sample side by side and compared the fragmentation patterns and their LC retention time. We were able to verify six metabolites against their standards, experimentally. Table V shows a list of the six verified and two unverified metabolites, and Fig. 3 shows an example of the verification result for $\mathrm{N}$-acetylDL-tryptophan, comparing the fragmentation pattern of the analyte in ductal lavage against the pattern obtained from an authentic compound.

\section{Discussion}

The emerging technology of metabolomics has provided insights into cancer metabolic pathways in different organs, including the mammary gland, which can lead to the discovery of new cancer biomarkers and therapies. To the best of our knowledge, this is the first study that compares the metabolomic profiles in the ductal fluid from cancer affected breasts 
vs. non-affected contralateral control breasts in the same subjects, allowing us to view the metabolites in the tumor microenvironment where breast cancer arises, in a unique well-controlled setting where each patient serves as her own control.

We analyzed ductal fluid samples obtained by ductal lavage from 43 subjects with unilateral breast cancer from both the affected breast and the contralateral non-affected control breast and identified 209 ions that showed a significant difference in intensity levels (q-values $<0.05$ ) between the fluid from the affected breast vs. the fluid from the non-affected control breast. We observed significant differences between the fluid from the affected breast vs. the fluid from the nonaffected control breast in the levels of tryptophan, products of lipid metabolism, derivatives of amino acid metabolism and phospholipids.

Interestingly, metabolomic profiles are not only different between cancer and normal tissue as we and others have shown, but also are cancer tissue type specific $(16,35)$. In addition, specific metabolite changes occur at earlier stages for each tissue and cancer type. For instance, amino acid related metabolites, such as N-Acetyl-amino acids, and lipid related metabolites, such as carnitine and glycerophopsholipids, were shown to be significantly altered in breast tumors $(14,16)$; in serum, amino acid changes were mainly observed in early stage breast cancer (stages I and II) compared to metastatic disease (14). A supervised class comparison analysis of tumor vs. non-tumor tissues revealed specific metabolites that could discriminate between liver, pancreas and breast, with the most evident differences occurring in amino acid and lipid pathways; glycerol and linolenate were upregulated only in breast tumors (16). In individuals with early stage disease, 8 metabolites were significantly and uniquely altered in breast tumors vs. normal tissues; in a similar analysis, 81 metabolites in liver and 18 in pancreatic tumors were significantly and differentially altered (16). Comparing the metabolic changes between tissue specific cancer and normal cells could pinpoint the metabolic reprograming involved in tissue specific tumorigenesis.

In the present study we identified lower levels of N-AcetylDL-tryptophan in the fluid from the affected breasts compared to the control breast fluid, consistent with previous reports showing decreased amino acid metabolites in breast cancer (36-38). Cancer metabolism requires energy derived from both anaerobic and aerobic glycolysis, which means lower substrate levels like glucose and glutamine being siphoned off to the tricarboxylic acid (TCA) cycle, and higher levels of bioenergic substrates, like lactate, which stimulates continuous growth (36). In a recent study, Willmann et al (39), used metabolomic profiling to sub-classify different breast cancer cell lines and differentiate not only between them, but also between the cancer cell lines and a non-cancer breast epithelial cell line as well. Research has shown that elevated glycine and lactate indicate increased metabolic changes due to rapid growth of cancer cells, and hence poorer prognosis (5).

Levels of metabolites were also reported to differ by race (38). However, in this study, we were not able to detect a statistically significant difference in the metabolite levels based on the subjects' race possibly due to the small sample size per each race. None of the LASSO-identified 21 ions showed statistically significant difference among patients based on race (minimum P-values $>0.50$; data not shown).

When we stratified our patients by receptor status, we observed that the M239.1486 metabolite tends to be less expressed in $\mathrm{ER}^{+}$patients $(\mathrm{P}=0.045$; Table IV). Fan et al (35) analyzed plasma metabolite profiles of 96 breast cancer patients compared to 79 normal controls by UPLC-Q/TOF-MS and $\mathrm{GC}-\mathrm{Q} / \mathrm{MS}$, and identified several metabolites that could discriminate between breast cancer and controls, and also between breast cancer subtypes according to HER2 and estrogen receptor status. ER positive $\left(E R^{+}\right)$patients showed elevated alanine, aspartate and glutamate metabolism, decreased glycerolipid catabolism and enhanced purine metabolism compared to the ER negative (ER) group (also described in plasma metabolites from breast cancer patients vs. normal controls (38). ER $\mathrm{ER}^{-}$and triple-negative receptor status is indicative of more aggressive tumors and a poorer prognosis. In breast cancer, the substrate glutamine and end product lactate enhance cancer aggressiveness; additionally $\mathrm{ER}^{-}$breast cancers are dependent upon serine synthesis for continued growth (36).

In the present study, metabolites involved in lipid signaling were decreased in the fluid from the breasts with tumors, similar to previously published study (36). Lower levels of lysophosphatidylcholines and higher levels of sphingomyelins and acylcarnitines were detected in the plasma of cancer patients compared to controls (37). The decrease in glycerophopsholipid levels in cancer patients may be due to a higher expression of phospholipase A2 (PLA2), a gene encoding for the enzymes responsible for their metabolism (37), and whose expression was found to be upregulated in breast cancer cell lines and tissues $(40,41)$. Higher levels of lipid metabolites, including fatty acids and carnitine metabolites, have been found in breast cancer patients compared to normal controls $(35,38,42)$. Wang et al $(9)$ reported that several lipids including phosphatidylglycerol (PG) were upregulated in highly invasive breast cancer cells. Breast tumors are described as developing a 'lipogenic phenotype', and while cell growth is dependent on lipogenesis, lipolysis is upregulated, and is associated with tumor aggressiveness $(36,37)$.

Although our findings do not show exactly the same metabolites being altered as reported in other studies, the metabolites that we identified belong to the same class of compounds and same metabolic pathways, especially in the case of lipid metabolites. Cancer cells have been shown to have an increased glucose uptake (Warburg effect), conversion to lactate (glycolysis) and changes in protein and lipid metabolism (43). Some differences between studies can be attributed to the biological specimens analyzed (ductal lavage) vs. tissue and blood, or to technical or methodological differences. Moreover, a large part of the differentially expressed metabolites from the present study are still unknown, emphasizing the complexity of metabolic alterations in cancer.

Because the ductal lavage samples were collected under anesthesia immediately before surgery when the patient was already medicated, we identified many drug-related metabolites in our samples. However, because many of these drug-related metabolites were different between the breasts with tumors and normal breasts, this altered drug metabolism in the affected breast could be of possible therapeutic 
benefit and requires additional analysis. Identification of the specific metabolites could be used to target specific enzymes. Enzyme expression levels and metabolic pathways could be used to target specific breast cancer phenotypes, or cancer cell survival within tumors (7).

A metabolomics based profile for the early detection of breast cancer recurrence has been developed by Asiago et al (15). They used 11 metabolites in their prediction model, several of which we have reported as being significantly differentially expressed in the ductal lavage samples. A follow-up analysis of our patients might yield comparable results and contribute to improving earlier detection and better treatment in the cases of breast cancer recurrence.

Although our study design was unique in that we used ductal fluid obtained from the contralateral normal breast for comparison, which allowed us to control for several variables that could not have been controlled for otherwise, a drawback of such a design is the possibility that the contralateral breast cells may already harbor metabolomic changes. We, and others, have shown that genomic aberrations occur very early in tumorigenesis and may precede morphologic changes (20,44-46). Therefore, we cannot rule out the possibility of similar changes affecting some metabolites in the contralateral clinically normal breast. A future study may include an additional control group of normal subjects (e.g., patients undergoing reduction mammoplasty) to account for this possibility.

In summary, this is the first study to assess metabolomic profiles in ductal fluid samples from breast cancer patients. The differences we saw were directly related to the tumor microenvironment and provide a snapshot of ongoing breast cancer metabolism. However, the identification of several metabolites with altered levels in the cancer affected breast, especially lipid related metabolites, previously reported in breast cancer tissue, cell lines and the circulation, provide confidence that these could constitute the basis for metabolomics based markers for breast cancer detection. This study shows the feasibility of conducting a comprehensive metabolomic profiling of breast tumors using breast ductal fluid to detect changes in the cellular microenvironment of the tumors and shows the potential for this approach to be used to improve detection of breast cancer.

\section{Acknowledgements}

The present study was supported by a grant from the Avon Foundation for Women.

\section{References}

1. American Cancer Society: Global Cancer Facts and Figures. 3rd edition. American Cancer Society, Atlanta, GA, 2015.

2. Warburg O: On the origin of cancer cells. Science 123: 309-314, 1956.

3. Denkert C, Bucher E, Hilvo M, Salek R, Orešič M, Griffin J, Brockmöller S, Klauschen F, Loibl S, Barupal DK, et al: Metabolomics of human breast cancer: New approaches for tumor typing and biomarker discovery. Genome Med 4: 37, 2012

4. Sengupta D and Pratx G: Imaging metabolic heterogeneity in cancer. Mol Cancer 15: 4, 2016.

5. Long JP, Li XN and Zhang F: Targeting metabolism in breast cancer: How far we can go? World J Clin Oncol 7: 122-130, 2016.
6. Mannello F and Ligi D: Resolving breast cancer heterogeneity by searching reliable protein cancer biomarkers in the breast fluid secretome. BMC Cancer 13: 344, 2013.

7. Shajahan-Haq AN, Cheema MS and Clarke R: Application of metabolomics in drug resistant breast cancer research. Metabolites 5: 100-118, 2015.

8. Claudino WM, Quattrone A, Biganzoli L, Pestrin M, Bertini I and Di Leo A: Metabolomics: Available results, current research projects in breast cancer, and future applications. J Clin Oncol 25: 2840-2846, 2007.

9. Wang J, Zuo Y, Man YG, Avital I, Stojadinovic A, Liu M, Yang X, Varghese RS, Tadesse MG and Ressom HW: Pathway and network approaches for identification of cancer signature markers from omics data. J Cancer 6: 54-65, 2015.

10. Sitter B, Lundgren S, Bathen TF, Halgunset J, Fjosne HE and Gribbestad IS: Comparison of HR MAS MR spectroscopic profiles of breast cancer tissue with clinical parameters. NMR Biomed 19: 30-40, 2006.

11. Mountford CE, Somorjai RL, Malycha P, Gluch L, Lean C, Russell P, Barraclough B, Gillett D, Himmelreich U, Dolenko B, et al: Diagnosis and prognosis of breast cancer by magnetic resonance spectroscopy of fine-needle aspirates analysed using a statistical classification strategy. Br J Surg 88: 1234-1240, 2001.

12. Budczies J, Denkert C, Müller BM, Brockmöller SF, Klauschen F, Györffy B, Dietel M, Richter-Ehrenstein C, Marten U, Salek RM, et al: Remodeling of central metabolism in invasive breast cancer compared to normal breast tissue - a GC-TOFMS based metabolomics study. BMC Genomics 13: 334, 2012.

13. Budczies J, Brockmöller SF, Müller BM, Barupal DK, RichterEhrenstein C, Kleine-Tebbe A, Griffin JL, Orešič M, Dietel M, Denkert C, et al: Comparative metabolomics of estrogen receptor positive and estrogen receptor negative breast cancer: Alterations in glutamine and beta-alanine metabolism. J Proteomics 94: 279-288, 2013

14. Jobard E, Pontoizeau C, Blaise BJ, Bachelot T, Elena-Herrmann B and Trédan O: A serum nuclear magnetic resonance-based metabolomic signature of advanced metastatic human breast cancer. Cancer Lett 343: 33-41, 2014.

15. Asiago VM, Alvarado LZ, Shanaiah N, Gowda GA, OwusuSarfo K, Ballas RA and Raftery D: Early detection of recurrent breast cancer using metabolite profiling. Cancer Res 70: 8309-8318, 2010.

16. Budhu A, Terunuma A, Zhang G, Hussain SP, Ambs S and Wang XW: Metabolic profiles are principally different between cancers of the liver, pancreas and breast. Int J Biol Sci 10: 966-972, 2014.

17. Armitage EG and Barbas C: Metabolomics in cancer biomarker discovery: Current trends and future perspectives. J Pharm Biomed Anal 87: 1-11, 2014.

18. Tredwell GD, Miller JA, Chow HH, Thompson PA and Keun HC: Metabolomic characterization of nipple aspirate fluid by ${ }^{1} \mathrm{H}$ NMR spectroscopy and GC-MS. J Proteome Res 13: 883-889, 2014.

19. Do Canto LM, Marian C, Willey S, Sidawy M, Da Cunha PA, Rone JD, Li X, Gusev Y and Haddad BR: MicroRNA analysis of breast ductal fluid in breast cancer patients. Int J Oncol 48: 2071-2078, 2016.

20. Isaacs C, Cavalli LR, Cohen Y, Pennanen M, Shankar LK, Freedman M, Singh B, Liu M, Gallagher A, Rone JD, et al: Detection of LOH and mitochondrial DNA alterations in ductal lavage and nipple aspirate fluids from hngh-risk patients. Breast Cancer Res Treat 84: 99-105, 2004.

21. Masood S: Development of a novel approach for breast cancer prediction and early detection using minimally invasive procedures and molecular analysis: How cytomorphology became a breast cancer risk predictor. Breast J 21: 82-96, 2015.

22. Sheikh KD, Khanna S, Byers SW, Fornace A Jr and Cheema AK: Small molecule metabolite extraction strategy for improving LC/ MS detection of cancer cell metabolome. J Biomol Tech 22: 1-4, 2011.

23. Kaur P, Rizk N, Ibrahim S, Luo Y, Younes N, Perry B, Dennis K, Zirie M, Luta G and Cheema AK: Quantitative metabolomic and lipidomic profiling reveals aberrant amino acid metabolism in type 2 diabetes. Mol Biosyst 9: 307-317, 2013.

24. Patterson AD, Li H, Eichler GS, Krausz KW, Weinstein JN, Fornace AJ Jr, Gonzalez FJ and Idle JR: UPLC-ESI-TOFMSbased metabolomics and gene expression dynamics inspector self-organizing metabolomic maps as tools for understanding the cellular response to ionizing radiation. Anal Chem 80: 665-674, 2008 . 
25. Kuhl C, Tautenhahn R, Böttcher C, Larson TR and Neumann S: CAMERA: An integrated strategy for compound spectra extraction and annotation of liquid chromatography/mass spectrometry data sets. Anal Chem 84: 283-289, 2012.

26. Storey J: False discovery rates. In: International Encyclopedia of Statistical Science. 1st edition. Lovric M (ed). Springer, p1673, 2011.

27. Westerhuis JA, van Velzen EJ, Hoefsloot HC and Smilde AK: Multivariate paired data analysis: Multilevel PLSDA versus OPLSDA. Metabolomics 6: 119-128, 2010.

28. Friedman J, Hastie T and Tibshirani R: Regularization paths for generalized linear models via coordinate descent. J Stat Softw 33: 1-22, 2010.

29. Reid S and Tibshirani R: Regularization paths for conditional logistic regression: The clogitL1 package. J Stat Softw 58: 12, 2014.

30. Zhou B, Wang J and Ressom HW: MetaboSearch: Tool for massbased metabolite identification using multiple databases. PLoS One 7: e40096, 2012.

31. Wishart DS, Knox C, Guo AC, Eisner R, Young N, Gautam B, Hau DD, Psychogios N, Dong E, Bouatra S, et al: HMDB: A knowledgebase for the human metabolome. Nucleic Acids Res 37 (Database): D603-D610, 2009.

32. Smith CA, O'Maille G, Want EJ, Qin C, Trauger SA, Brandon TR, Custodio DE, Abagyan R and Siuzdak G: METLIN: A metabolite mass spectral database. Ther Drug Monit 27: 747-751, 2005.

33. Cui Q, Lewis IA, Hegeman AD, Anderson ME, Li J, Schulte CF, Westler WM, Eghbalnia HR, Sussman MR and Markley JL: Metabolite identification via the Madison Metabolomics Consortium Database. Nat Biotechnol 26: 162-164, 2008.

34. Sud M, Fahy E, Cotter D, Brown A, Dennis EA, Glass CK, Merrill AH Jr, Murphy RC, Raetz CR, Russell DW, et al: LMSD: LIPID MAPS structure database. Nucleic Acids Res 35 (Database): D527-D532, 2007.
35. Fan Y, Zhou X, Xia TS, Chen Z, Li J, Liu Q, Alolga RN, Chen Y, Lai MD, Li P, et al: Human plasma metabolomics for identifying differential metabolites and predicting molecular subtypes of breast cancer. Oncotarget 7: 9925-9938, 2016

36. Mishra P and Ambs S: Metabolic signatures of human breast cancer. Mol Cell Oncol 2: 2, 2015.

37. Qiu Y, Zhou B, Su M, Baxter S, Zheng X, Zhao X, Yen Y and Jia W: Mass spectrometry-based quantitative metabolomics revealed a distinct lipid profile in breast cancer patients. Int J Mol Sci 14: 8047-8061, 2013.

38. Shen J, Yan L, Liu S, Ambrosone CB and Zhao H: Plasma metabolomic profiles in breast cancer patients and healthy controls: By race and tumor receptor subtypes. Transl Oncol 6: 757-765, 2013

39. Willmann L, Schlimpert M, Halbach S, Erbes T, Stickeler E and Kammerer B: Metabolic profiling of breast cancer: Differences in central metabolism between subtypes of breast cancer cell lines. J Chromatogr B Analyt Technol Biomed Life Sci 1000: 95-104, 2015.

40. Yamashita J, Ogawa M and Sakai K: Prognostic significance of three novel biologic factors in a clinical trial of adjuvant therapy for node-negative breast cancer. Surgery 117: 601-608, 1995.

41. Yamashita S, Yamashita J, Sakamoto K, Inada K, Nakashima Y, Murata K, Saishoji T, Nomura K and Ogawa M: Increased expression of membrane-associated phospholipase A2 shows malignant potential of human breast cancer cells. Cancer 71: 3058-3064, 1993.

42. Takayama T, Tsutsui H, Shimizu I, Toyama T, Yoshimoto N, Endo Y, Inoue K, Todoroki K, Min JZ, Mizuno H, et al: Diagnostic approach to breast cancer patients based on target metabolomics in saliva by liquid chromatography with tandem mass spectrometry. Clin Chim Acta 452: 18-26, 2016.

43. Hadi NI and Jamal Q: 'OMIC' tumor markers for breast cancer: A review. Pak J Med Sci 31: 1256-1262, 2015. 